\title{
OBSERVATIONS
}

\section{SUR L'HÉRÉDITÉ DE LA CARPE (1)}

\author{
par le Docteur-Ingénieur I. POJOGA
}

Le poisson a une grande importance pour l'alimentation de l'homme et un peu moins pour celle des animaux, grâce à l'utilisation de la farine de poissons.

La composition chimique de la chair du poisson a autant de valeur que celle des bovins, des porcins et des oiseaux de basse-cour; elle se montre parfois même supérieure. Ainsi l'anguille avec 29 à $37 \%$ de graisse, le silure avec $37 \%$, se rangent à côté du porc.

Le brochet a de 19 à $20 \%$ de protèines, le gardon $19,63 \%$; le silure $21 \%$, la truite $19,56 \%$, la carpe $17,3 \%$ alors que les bovins en dosent $21 \%$. les oies $17 \%$ et les porcs $15 \%$.

La valeur énergétique en calories est élevée et le degré d'assimilation considérable atteignant $96 \%(74-76 \%$ chez les bovins). Les vitamines $\mathrm{A}$ et $B_{12}$ n'existent que chez les animaux et cette dernière facilite l'assimilation des protéines végétales.

Dans la plupart des pays du monde, le développement de la pisciculture intensive a pris une grande importance et pose des problemes qui devraient ètre résolus.

Parmi de nombreuses recherches dans le domaine de l'hydrobiologie

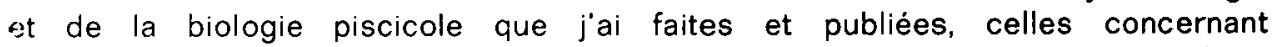
T'hérédité de la carpe ont un grand intérêt scientifique et pratique, la pisciculture etant une branche de la zootechnie (fig. 1).

(1) Résumé d'un exposé présenté au Congrès de l'Union régionale des Fédérations departementales des Associations agrées de pêche et de pisciculture des bassins de la Garonne. de l'Adour et de la Charente (Dax, mai 1969).

Article available at http://www.kmae-journal.org or http://dx.doi.org/10.1051/kmae:1969002 
Le choix des reproducteurs, le croisement des différentes races en vue de l'obtention d'un cheptel piscicole ayant un rythme de croissance superieur et une plus grande résistance aux maladies contribuent directement à l'augmentation de la production piscicole et par voie de conséquence, à l'élévation du niveau de l'alimentation humaine.

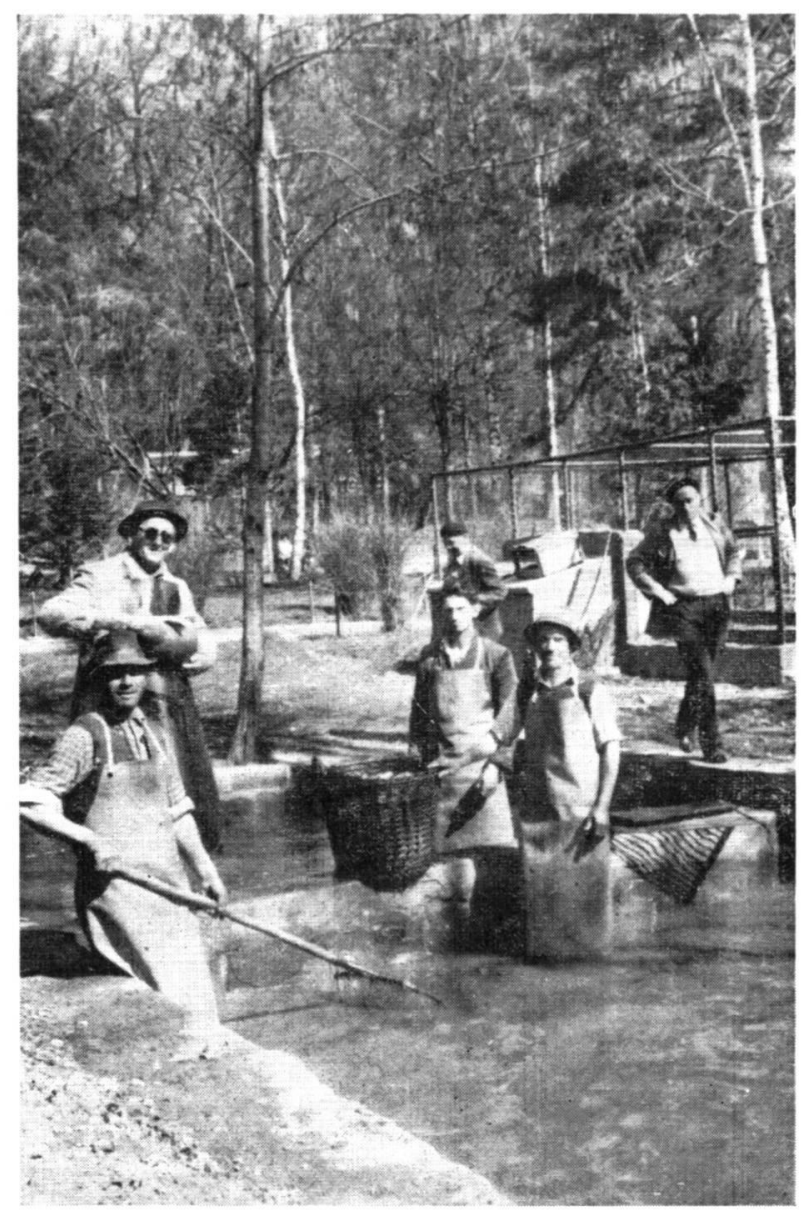

Photo J. Pogoda.

Fig. 1 - Station de carpiculture "DUMBRAVA SIBIU • (ROUMANIE). Travaux de Sélection de Carpe Olt.

L'hérédité détermine le caractère spécifique des processus biologiques des êtres vivants en liaison avec le milieu ambiant. De là, l'apparition de certaines particularités morphologiques et physiologiques qui se transmettent de générations en générations. Dans un milieu donné, l'organisme n'assimile pas n'importe quel élément mais seulement ceux spécifiques à leur hérédité (FURTUNESCU) (6).

Cette observation générale trouve son application dans l'élevage de la carpe. 
KIRPICNIKOV (8) répartit les carpes en quatre sous-espèces :

1 - Europe et Trans-caucase : Cyprinus carpio.

2 - Asie centrale : Cyprinus carpio aralensis.

3 - Amour et Chine: Cyprinus carpio haecmatopterus.

4 - Vietnam : Cyprinus carpio viridiviolacens.

Ces sous-espèces diffèrent par le nombre des rayons des nageoires dorsales, des branchiospines et des écailles le long de la ligne latérale, par la grandeur des yeux et la longueur des barbillons.

Les monstruosités craniennes, particulièrement chez la carpe, ont surtout intéressé les auteurs. BONAPARTE (2) cite un barbeau (Barbus barbus) présentanî une déformation telle qu'il ressemblait à une carpe. LANDOIS (10) décrit un chevaine mops (Squalius dobula) et LUNEL (11) un vairon (Phoxinus phoxinus), CANESTRINI (3) chez le muge doré (Mugil liza auratus), FEDDERSEN (5) chez le Gadus morrhua, YARELL (17) chez la truite commune (Salmo trutta), VROLIK chez le brochet, VAN LIDTH DE JEUDE (18) chez le silure (Silurus glanis), ANTIPA (1) chez le Sandre (Lucioperca sander), la brême (Abramis brama) (fig. 2), l'orfe, (Idus melanotus), l'ablette (Alburnus lucidus) et même chez l'esturgeon.

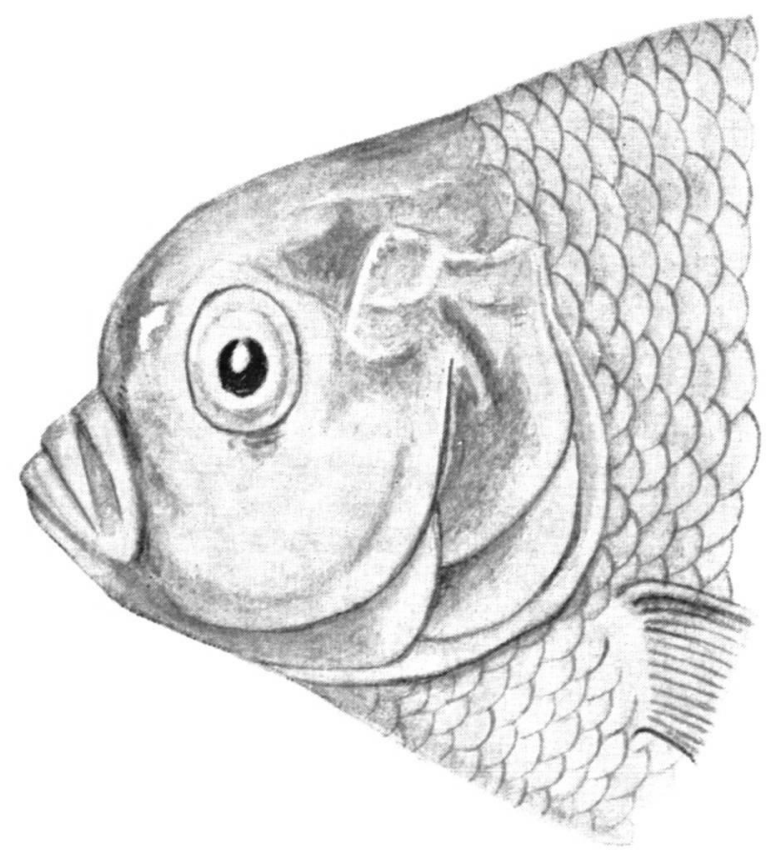

Fig. 2 - BREME *Abramis brama $L$ ". Monstruosité céphalique "Box".

Parmi un grand nombre de types rencontrés après la guerre en Roumanie se trouve la carpe dite *box" (fig. 3 et 4) ou à tête de dauphin (Cyprinus carpio var. caput delphini) (Fig. 3 et 4). NEYDECH (12) croyait que c'était probablement un hybride de carpe et de barbeau, STEINDACHNER et VALENCIENNES (4) estimaient que c'était une déformation congénitale. KNAUTHE (9) affirme que cette monstruositè est héréditaire. 
Ne connaissant pas les recherches de KNAUTHE, j'ai fait à la Faculté d'Agronomie Chisineff de l'Université de lassy das les bassins expérimentaux des laboratoires d'hydrobiologie et de pisciculture les croisements suivants

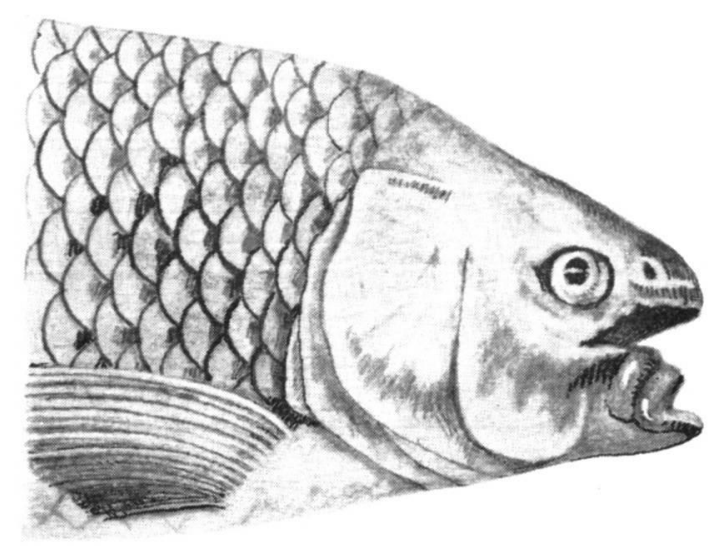

Fig. 3 - CARPE "Cyprinus Carpio L». Monstruosité céphalique «BOX ".

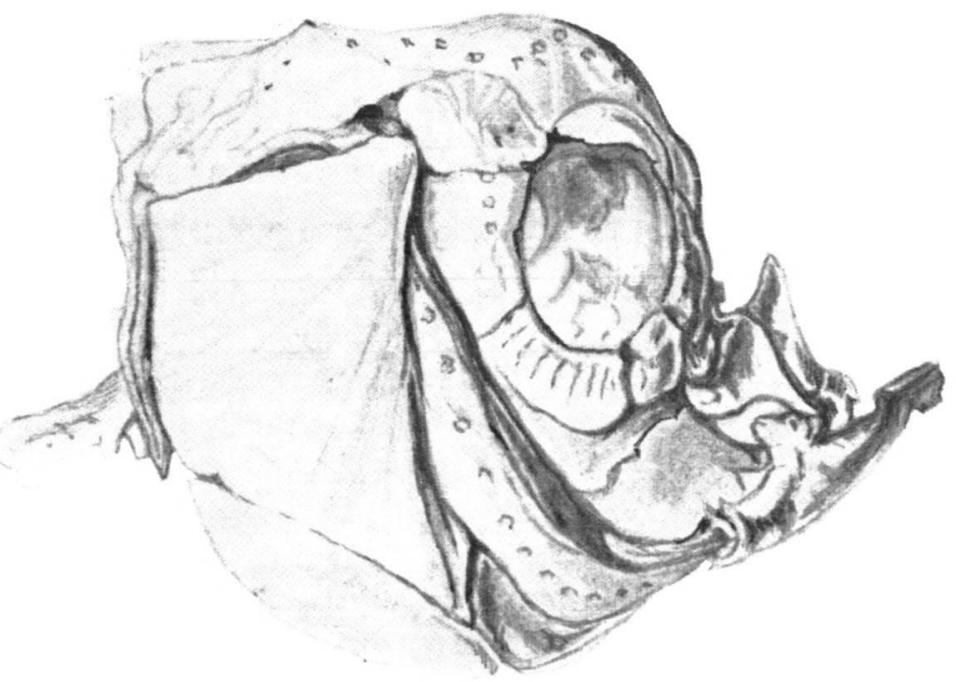

Fig. 4 - Crâne de Carpe "Box". Vue de profil. Monstruosité céphalique. 
1 - Carpe galicienne femelle $\times$ carpe var. caput delphini (provenant du Nistre) mâle : Résultats $87 \%$ carpes à écailles (dont $76 \%$ à tête * box ") et $13 \%$ du type galicien c'est-à-dire * miroir ".

2 - Carpe de Lausitz femelle $\times$ carpe var. caput delphini mâle Résultats : carpes à écailles dont $62 \%$ à tête "box".

3 - Carpe "box" femelle $\times$ carpe "box " mâle - Résultats : carpes à écailles dont $92 \%$ avec le squelette de la tête déformé.

On constate que la carpe à tête de dauphin (C. carpio caput delphini) est une variété stable, ancienne dont les caractères génétiques sont dominants. Cette varièté de carpe a disparu dans les dernières dizaines d'années, à la suite de pêches intensives et du dessèchement de grandes masses d'eau stagnantes en vue d'une distribution à l'agriculture. Cependant nous avons rencontré des exemplaires de carpe "box" dans beaucoup d'élevages où la présence de cette variété n'avait pas été signalée.

N'ayant pas continué nos recherches à la Faculté d'Agronomie par force majeure, nous les avons reprises à la Station de sélection de "Dumbrava Sibiu " de 1954 à 1962, dans le but de déterminer les caractères morphologiques el physiologiques imprimés par les géniteurs à la descendance.

A partir de 1956, nos observations ont été poursuivies dans les 3 étangs de reproduction ci-dessous :

L'étang no 1, d'une surface de $3600 \mathrm{~m}^{2}$ a été peuplé de carpes femelles galiciennes et de carpes mâles de Lausitz dont les caractéristiques étaient les suivantes :

\begin{tabular}{|c|l|l|l|l|l|l|}
\hline Race & $\begin{array}{c}\mathrm{L} \\
\mathrm{cm}\end{array}$ & $\begin{array}{c}\mathrm{I} \\
\mathrm{cm}\end{array}$ & $\begin{array}{c}\mathrm{H} \\
\mathrm{cm}\end{array}$ & $\begin{array}{c}\mathrm{l} / \mathrm{H} \\
\mathrm{cm}\end{array}$ & $\begin{array}{c}\text { Age } \\
\text { ans }\end{array}$ & $\begin{array}{c}\text { Poids } \\
\text { en } \mathrm{gr}\end{array}$ \\
\cline { 1 - 5 } & 56 & 47 & 17 & 2,7 & $4+$ & 2000 \\
Galicienne & 56,5 & 44 & 16 & 2,7 & $4+$ & 1900 \\
$:$ & 52,5 & 60 & 21 & 2,8 & $7+$ & 5000 \\
$:$ & 39 & 32 & 12 & 2,6 & 3 & 1000 \\
Lausitz & 67 & 59 & 21 & 2,8 & 7 & 5500 \\
$:$ & 54 & 48 & 17 & 2,8 & 4 & 1950 \\
$:$ & 67,5 & 59 & 20 & 2,9 & 7 & 5400 \\
$:$ & 44,5 & 38 & 14 & 2,7 & 4 & 1500 \\
\hline
\end{tabular}

La reproduction a eu lieu du 5 au 30 juin. En août les alevins ont été placés dans des étangs d'élevage à raison de 20000 par ha. En automne, ceux-ci d'un poids moyen de $36 \mathrm{gr}$ appartenaient pour $20 \%$ à la race galicienne et $80 \%$ à la race de Lausitz. Les mâles semblent donc övoir imprimé les caractères dominants.

Voici que!ques caracteristiques de ces alevins :

\begin{tabular}{|c|c|c|c|c|c|}
\hline Race & $\begin{array}{c}\mathrm{L} \\
\mathrm{cm}\end{array}$ & $\begin{array}{c}\mathrm{I} \\
\mathrm{cm}\end{array}$ & $\begin{array}{c}\mathrm{H} \\
\mathrm{cm}\end{array}$ & $\begin{array}{c}\mathrm{I} / \mathrm{H} \\
\mathrm{cm}\end{array}$ & $\begin{array}{c}\text { Poids } \\
\mathrm{gr}\end{array}$ \\
\cline { 2 - 6 } & 18 & 14 & 6,5 & 2,3 & 60 \\
Lausitz & 10,5 & 8,5 & 3,8 & 2,3 & 18 \\
Galicienne & 14,5 & 12 & 3,5 & 2,5 & 50 \\
$*$ & 14,5 & 7 & 2,5 & 2,3 & 10 \\
\hline
\end{tabular}


L'ètang de reproduction nc 2 d'une surface de $2500 \mathrm{~m}^{2}$ a èté peuplé exclusivement de carpes mâles et femelles Lausitz dont les caractéristiques sont données ci-dessous

\begin{tabular}{|c|c|c|c|c|c|c|c|}
\hline Race & $\begin{array}{c}\mathrm{L} \\
\mathrm{cm}\end{array}$ & $\begin{array}{c}1 \\
\mathrm{~cm}\end{array}$ & $\begin{array}{c}\mathrm{H} \\
\mathrm{cm}\end{array}$ & $\begin{array}{l}\mathrm{H} / \mathrm{l} \\
\mathrm{cm}\end{array}$ & $\begin{array}{c}\text { Age } \\
\text { ans }\end{array}$ & $\begin{array}{l}\text { Poids } \\
\text { en } \mathrm{gr}\end{array}$ & Sexe \\
\hline Lausitz & 34 & 36 & 14,5 & 2,9 & 4 & 1500 & Mâles \\
\hline . & 37,5 & 32 & 11.5 & 2,8 & 3 & 1000 & $"$ \\
\hline . & 39 & 32,5 & 12 & 2,7 & 3 & 1100 & . \\
\hline . & 58 & 49,5 & 18 & 2.7 & 6 & 3500 & • \\
\hline , & 66 & 57 & 19,5 & 2,9 & 7 & 6000 & $"$ \\
\hline . & 66 & 59 & 20 & 2,9 & 7 & 6150 & femelles \\
\hline . & 65,5 & 52 & 18 & 2,9 & 6 & 4000 & $\bowtie$ \\
\hline$=$ & 71 & 61 & 22 & 2,7 & 7 & 7000 & $"$ \\
\hline * & 60,5 & 52 & 22,5 & 2,3 & 6 & 6000 & $"$ \\
\hline
\end{tabular}

La reproduction a eu lieu du 6 au 25 mai, donc beaucoup plus tôt que dans l'étang no 1. L'exposition de l'étang était plus favorable et les sujets appartenaient à la même race.

En aoint les alevins ont été, comme dans l'étang no 1, placés à raison de 20000 par ha. dans un étang d'élevage. Ils pesaient en automne un poids moyen de $35 \mathrm{gr} .86 \%$ d'entre eux appartenaient à la race Lausitz, et $14 \%$ à la race galicienne, c'esì-à-dire du type "miroir", ce qui montre que les reproducteurs Lausitz utilisés par nous, en Roumanie, ne constituent pas une race pure. Voici quelques caractéristiques de ces alevins:

\begin{tabular}{|c|c|c|c|c|c|}
\hline Race & $\begin{array}{c}\mathrm{L} \\
\mathrm{cm}\end{array}$ & $\begin{array}{c}\mathrm{I} \\
\mathrm{cm}\end{array}$ & $\begin{array}{c}\mathrm{H} \\
\mathrm{cm}\end{array}$ & $\begin{array}{c}\mathrm{I} / \mathrm{H} \\
\mathrm{cm}\end{array}$ & $\begin{array}{c}\text { Poids } \\
\mathrm{gr}\end{array}$ \\
\hline Lausitz & 17 & 14 & 6,3 & 2,3 & 100 \\
$\cdots$ & 12,5 & 10,3 & 4,5 & 2,2 & 22 \\
$\cdots$ & 10,0 & 8,5 & 3 & 2,1 & 20 \\
$\cdots$ & 21,0 & 17,5 & 7 & 2,3 & 130 \\
Galicienne & 16,5 & 13,5 & 4 & 2,4 & 70 \\
$\cdots$ & 13,5 & 11,3 & 4 & 2,4 & 35 \\
\hline
\end{tabular}

L'étang de reproduction no 3 , d'une superficie de $3500 \mathrm{~m}^{2}$ était peuplé de carpes sauvages mâles du Olt et de carpes Lausitz femelles dont les caractéristiques étaient les suivantes :

\begin{tabular}{|c|c|c|c|c|c|c|}
\hline Race & $\underset{\mathrm{cm}}{\mathrm{L}}$ & $\begin{array}{c}\mathrm{l} \\
\mathrm{cm}\end{array}$ & $\begin{array}{l}\mathrm{H} \\
\mathrm{cm}\end{array}$ & $\begin{array}{l}1 / \mathrm{H} \\
\mathrm{cm}\end{array}$ & $\begin{array}{l}\text { Age } \\
\text { ans }\end{array}$ & $\begin{array}{c}\text { Poids } \\
\text { gr }\end{array}$ \\
\hline $\begin{array}{c}\text { Carpe du Olt } \\
\vdots \\
\text { Lausitz } \\
\vdots \\
\end{array}$ & $\begin{array}{l}54 \\
47 \\
31 \\
45,5 \\
52 \\
54 \\
73 \\
65 \\
41\end{array}$ & $\begin{array}{l}56 \\
40,5 \\
35 \\
38 \\
44,5 \\
47 \\
64 \\
55 \\
35\end{array}$ & $\begin{array}{l}13 \\
12,5 \\
11 \\
12 \\
13,5 \\
12 \\
22,5 \\
18 \\
12\end{array}$ & $\begin{array}{l}3,5 \\
3,2 \\
3,1 \\
3,1 \\
3,2 \\
3,1 \\
2,8 \\
2,9 \\
2,9\end{array}$ & $\begin{array}{l}4+ \\
4+ \\
3+ \\
3 \\
5 \\
4 \\
6 \\
6 \\
3\end{array}$ & $\begin{array}{l}2000 \\
1700 \\
1000 \\
1400 \\
2000 \\
1500 \\
6000 \\
5000 \\
1600\end{array}$ \\
\hline
\end{tabular}


En automne, les alevins ètaient pour $99,5 \%$ à écailles; $0,5 \%$ seulement d'entre eux avaient les caractères de la carpe miroir, ce qui, la carpe Lausitz étant à écailles, semble indiquer d'une part que cette race n'est pas pure et que d'autre part la carpe sauvage du Olt, d'origine plus ancienne, a transmis de préférence ses caractères dominants. C'est une race stable.

\section{CONCLUSIONS}

II semble donc que l'on puisse faire les conclusions suivantes :

1 - Les races pures de carpes les plus anciennes ont la propriété d'imprimer à leurs descendants les caractères dominants.

2 - Lorsqu'il s'agira de sélectionner des sujets en vue d'obtenir par croisements des races nouvelles de productivité supérieure, il faudra utiliser les formes ies plus anciennes.

3 - II en sera de même en vue de l'obtention de races résistantes à certaines maladies épidémiques.

4 - L'apparition de la race "box" doit être considérée comme une mutation spontanée.

5 - Les carpes des eaux sauvages et les carpes d'origine ancienne doivent être protégées, comme on le fait pour d'autres vertébrés.

\section{B I BLIOGRAPHIE}

1 ANTIPA G.R., 1909, Fauna ichtiologica a României Academia Româna, editura Academia Româna, 294 p.

2 BONAPARTE, Fauna italica, III, Pesci.

3 CANESTRINI, 1889, Atte della soc. Ven. Trent. di scient. nat. in Padova, IX, $117 \mathrm{p}$.

4 CUVIER et VALENCIENNES, 1828-1849, Histoire naturelle des Poissons, XVI, $187 \mathrm{p}$.

5 FEDDERSEN A., Dansk Eisscerritidende

6 FURTUNESCU, 1965, Zootechnia generala, genetica, Bucuresti, 425 p.

7 BERG L.S., 1949, Ribi presnih vod SSSR. i sopredelenih stran. vol. II 925 p.

8 KIRPICNIKOV, 1962, Novie pod vidi carpa. U.R.S.S.

9 KNAUTHE, 1893, Biologisches Centralblatt.

10 LANDOIS, 1883, Zoologischer Garten, I. XXIV, 298 p.

11 LUNEL, Poissons du Lac Léman, $96 \mathrm{p}$.

12 NEYDECH, 1849, Jahrbuch des Mannheimer Verein. f. Naturkunde.

13 NIKOLSKII G.V., 1961, Ekologia Râb., Moskova.

1950, Ciastnasia ichtiologia Sovetskaia Nauka, 436 p.

14 POJOGA I., 1960, Piscicultura, Editura Adro, Silvica, 534 p.

15 SKATKIN, 1962, Biologhiceskie osnovi iskusstevennogo riborazvedenia, Academia Nauk S.S.S.R.

16 SUVOROV E.C., 1948, Osnovi ichtiologii 579 p.

17 YARELL, British Fishes I, 286 p.

18 VAN LIDTH DE JEUDE, Not. Leyden Museum, VII, 259 p.

19 VIVIER P., 1962, La Pisciculture, Presses Universitaires de France, Paris, 126 p. 


\section{ANALYSE BIBLIOGRAPHIQUE}

VIVIER P. - Pisciculture - In «Techniques Agricoles" (publication de l'Association pour le développement et la vulgarisation des techniciues agricoles), l, fasc. 3850 à 3854, 49 p. Paris, 1968.

Techniques agricoles, "encyclopédie des connaissances nécessaires à la gestion des entreprises agricoles", présente la particularité d'être publiée par fascicules détachables. On peut alors facilement les remplacer ou les modifier par de nouveaux fascicules ou des fiches intercalables fournis par l'éditeur, à mesure de la progression entièrement rapide de la science et de láa technique en agriculture, ce qui permet d'avoir ainsi une collection constamment tenue à jour.

Les professeurs Jean KEILLING et Jacques CASALIS, rédacteurs en chef de cette encyclopédie, ont estimé justement que la pisciculture devait y avoir sa place. Malgré le grand nombre d'associations de pêche et de pisciculture, le dynamisme du Syndicat national des pisciculteurs-salmoniculteurs et du Syndicat de l'Etang, la pisciculture n'est en France qu'une activité agricole secondaire : le nombre de pages à lui réserver dans cette encyclopédie ne pouvait être que restreint. C'est donc un condensé, inévitablement incomplet que l'Auteur a rédigé.

Dans une première partie consacrée au poisson et à son milieu, M. VIVIER, après avoir donné des renseignements succints sur le caractère juridique du poisson d'eau douce, sur son importance économique, sa reproduction, les principaux facteurs de sa croissance, definit en quelques mots la chaine alimentaire dont il est un des maillons extrêmes et qui revêt des aspects différents dans les lacs, les étangs et les cours d'eau.

La $2 \mathrm{e}$ partie de la publication, la plus importante, a trait aux principaux élevages : élevage des Salmonidés, de la truite surtout, élevage de la carpe, élevage des carnassiers de repeuplement, brochet, sandre, black-bass qui jouent un rôle important et nécessaire pour le maintien de l'équilibre biologique de nos cours d'eau et de nos étangs. L'auteur donne par de nombreuses illustrations des renseignements pratiques sur les installations modernes de pisciculture en étangs, bassins, digues, vidanges, pêcherie, appareils de triage, etc.

Les principes d'aménagement piscicoles forment la $3 e$ partie. L'Auteur traite de la productivité, des principaux facteurs qui la favorisent ou qui la défavorisent après avoir rappelé ce qu'est l'équilibre biologique dans les différents milieux aquatiques. Puis il aborde le problème capital des repeuplements artificiels, de leur justification dans certains cas et indique les différentes méthodes utilisées ; il rappelle les formules pratiques de détermination du nombre d'alevins à immerger, formules de LEGER, formules de HUET, formules de MARTIN et LECOINTRE. Il insiste sur la pratique du déversement d'alevins ou d'adultes, opération aussi délicate qu'essentielle et trop souvent sacrifiée dans la pratique. If donne enfin quelques renseignements succints sur les passes à poissons.

Bien qu'elle s'adresse plus spécialement aux agriculteurs, cette publication condensée et pratique doit rendre service aux pisciculteurs et aux associations de pêche et de pisciculture. 